\title{
Chiral symmetry and dileptons in heavy ion collisions
}

\author{
F. Karsch ${ }^{1,2}$, K. Redlich ${ }^{1,3}$, L. Turko ${ }^{3}$ \\ 1 Fakultät für Physik, Universität Bielefeld, D-33615 Bielefeld, Germany \\ ${ }^{2}$ HLRZ, Forschungszentrum Jülich, D-52425 Jülich, Germany \\ 3 Department of Theoretical Physics, University of Wrocław, PL-50-204 Wrocław, Poland
}

Received 12 March 1993; in revised form 7 May 1993

\begin{abstract}
We study the consequences of chiral symmetry restoration for thermal production of dileptons in central nucleus-nucleus collisions. We discuss the temperature dependence of various hadronic parameters entering the dilepton cross-section, putting emphasis on universal properties in the vicinity of a 2 nd order chiral symmetry restoring phase transition and the constraints on their temperature dependence known from lattice simulations. It is shown that the modifications of the hadron spectrum due to medium effects can lead to important changes of the thermal dilepton production rate in heavy ion collisions. In particular, it can imply the suppression of a thermal $\rho$ peak.
\end{abstract}

\section{Introduction}

It is generally expected that the hadron spectrum undergoes large qualitative changes in hadronic matter at finite temperature and/or density. Most of the low lying hadronic states are expected to be dissolved in the quark gluon plasma phase. However, also below the deconfining, phase transition modifications are expected due to the gradual melting of the chiral condensate. In fact, the properties of hadronic matter in the vicinity of the phase transition are expected to be controlled mainly by the universal structure of a chiral Lagrangian, which among other things will also control the temperature dependence of the chiral condensate. This will influence the temperature dependence of hadron masses and their decay widths.

Various phenomenological implications of the medium effects on the hadron spectrum have been discussed in the literature recently [1-6]. In this work we shall concentrate on thermal dilepton production, which is mainly affected by the temperature dependence of pion and $\rho$ meson masses and their decay widths. The behaviour of hadron parameters at finite $T$ has been studied recently within the framework of chiral perturbation theory [7], the virial expansion [8], a Nambu-Jona-Lasinio model [9], QCD sum rules [10] as well as lattice gauge theory [11]. These investigations lead to similar conclusions about the behaviour of the low lying hadron masses for temperatures well below the chiral symmetry restoration phase transition temperature. Lattice simulations in quenched QCD, as well as in the presence of light dynamical quarks, suggest that the quark condensate and hadron masses change only little for $T \lesssim 0.75 T_{c}$. This is in accordance with chiral perturbation theory and finite temperature sum rule results. In particular this latter approach also suggests a close link between the properties of hadrons composed of $u$ and $d$ quarks and the quark condensate in a medium, $\langle\bar{\Psi} \Psi\rangle_{T}[3,12]$. If this link between the chiral condensate and the hadron masses remains to be valid in the limit $T \rightarrow T_{c}$, then a strong variation of hadron masses close to $T_{c}$ is to be expected. In the case of a 2nd order chiral symmetry restoring phase transition, where the chiral condensate vanishes, it seems to be likely that also the rho meson mass will vanish at $T_{c}$ [13-15].

The change of the $\rho$ decay width with temperature will be related to the temperature dependence of the rho and pion masses. When assuming that both masses exhibit only a slow variation with temperature in the vicinity of $T_{c}$, the rho decay width $\Gamma_{\rho}$ will most likely increase when approaching $T_{c}$. However, if $\rho$ and/or $\pi$ mass decreases, then $\Gamma_{\rho}$, becomes most probably small because of the phase space factor.

It is obvious and, in fact, it has already been indicated in the literature $[1,6]$, that the variation of hadron masses and their decay widths with temperature will significantly influence the dilepton spectrum. The aim of our present work is to investigate the consequences of a second order chiral transition on the dilepton spectrum. In particular, we shall show, that independent of the concrete form of the temperature dependence chosen for hadronic parameters, the experimentally measured dilepton spectrum will be significantly modified. This modification is particularly evident in the vicinity of rho meson resonance in the dielectron invariant mass distribution.

The paper is organized as follows: In Sect. 2 we review some basic results about thermal dilepton production and 
introduce the relevant parameters. In Sect. 3 we shall discuss the variation of hadron parameters with temperature. In Sect. 4 we describe the dependence of the dilepton production rate on the time evolution of QCD matter and discuss our results. Finally we give our conclusions in Sect. 5 .

\section{Dilepton production in hot hadronic matter}

Since a long time dilepton production has been considered as an important observable, which allows to examine the structure of matter produced in ultra-relativistic heavy ion collisions [16-21]. As these are electromagnetically interacting particles, once produced, they will escape from the medium with no further interactions, thus transmitting information about the primordial state of matter produced in a collision to the observer.

Dileptons emerge from various sources in heavy ion collisions. While the low invariant mass dilepton pairs are predominantly produced in the decay of light mesons [18] or due to virtual photon bremsstrahlung [18-20], those with a large invariant mass are mainly due to Drell-Yan production. Furthermore, it is expected that hadronic matter, produced in heavy ion collisions, thermalizes and thus will also lead to thermal dilepton production through quark-antiquark pair annihilation in the quark-gluon plasma phase and pion annihilation in the hadronic phase. If the initial temperature of the medium is high enough, these thermal dileptons can be emitted for a long time and thus should dominate the spectrum [21]. We note, that this is even true, when we discuss phenomena which only influence the dilepton production in the hadronic phase, like the modification of hadronic resonance parameters due to chiral symmetry restoration; a large initial temperature leads to a large hadronic source after cooling and expansion of the initially produced medium.

The modification of hadronic parameters, of course, can only influence the dilepton production in the hadronic phase. In a hadron gas the cross section, $\sigma_{\pi}$, for pion annihilation, $\pi^{+} \pi^{-} \rightarrow \gamma^{*} \rightarrow L^{+} \ell^{-}$, can be calculated in analogy to the basic QED process, $e^{+} e^{-} \rightarrow L^{+} \ell^{-}$; the main difference is, that now vector meson resonance formation plays a crucial role. The cross section is given by the wellknown expression,

$\sigma_{\pi}(M)=\sigma(M)\left|F_{\pi}(M, T)\right|^{2}\left(1-\frac{4 m_{\pi}^{2}}{M^{2}}\right)^{1 / 2}$,

where $\sigma(M)$ denotes the basic QED cross section

$\sigma(M)=\frac{4 \pi}{3} \frac{\alpha^{2}}{M^{2}}\left(1+\frac{2 m_{\ell}^{2}}{M^{2}}\right)\left(1-\frac{4 m_{\ell}^{2}}{M^{2}}\right)^{1 / 2}$,

and $F_{\pi}$ is the pion form factor, which in the Breit-Wigner approximation, assuming the dominance of the $\rho$-resonance contribution, is given by

$\left|F_{\pi}(M, T)\right|^{2}=\frac{m_{\rho}^{4}+m_{\rho}^{2} \Gamma_{\rho}^{2}(T)}{\left(M^{2}-m_{\rho}^{2}\right)^{2}+m_{\rho}^{2} \Gamma_{\rho}^{2}(T)}$,

Here $m_{\rho}$ is the $\rho$ mass, and $\Gamma_{\rho}$ the $\rho \mapsto \pi \pi$ decay width, which at $T=0$ take on the values $m_{\rho}=775 \mathrm{MeV}$ and $\Gamma_{\rho}=155 \mathrm{MeV}$. We note that $F_{\pi}$ actually depends on the ratios $\Gamma_{\rho} / m_{\rho}$ and $M / m_{\rho}$. The cross section for thermal dilepton production due to pion annihilation will then depend crucially on the temperature dependence of these ratios. A decreasing $\rho$-mass will lead to a shift in the $\rho$-resonance peak, visible in the zero temperature spectrum, to smaller invariant masses. The temperature dependence of the ratio $\Gamma_{\rho} / m_{\rho}$ will control whether this resonance contribution will be enhanced or suppressed. A temperature dependent pion mass, on the other hand, will mainly lead to modifications of the phase space cut-off at low invariant mass.

The total dilepton invariant mass spectrum at temperature $T$ per unit space-time volume, $\mathrm{d} x^{4}$, is given by $[16,22]$

$$
\frac{\mathrm{d} N}{\mathrm{~d}^{4} \times \mathrm{d} M}=\frac{1}{(2 \pi)^{4}} M^{2} T^{2} \sigma_{\pi}\left(M^{2}\right)\left(M^{2}-4 m_{\pi}^{2}\right)^{1 / 2} G\left(T, M^{2}\right) .
$$

Here we have defined

$G=\int_{\lambda}^{\infty} \mathrm{d} x\left(\mathrm{e}^{x}-1\right)^{-1} \ln \left[\frac{1-\exp \left[-W x-P\left(x^{2}-\lambda^{2}\right)^{1 / 2}\right]}{1-\exp \left[-W x+P\left(x^{2}-\lambda^{2}\right)^{1 / 2}\right]}\right]$,

with

$W \equiv \frac{M^{2}}{2 m_{\pi}^{2}}-1 ; \quad \lambda \equiv \frac{m_{\pi}}{T} ; \quad P \equiv\left(W^{2}-1\right)^{1 / 2}$.

These equations form the basis for our analysis of dilepton spectra at finite temperature, which we are going to discuss in the following sections.

\section{Hadron parameters in the chiral limit}

The temperature dependence of $m_{\rho}$, as well as $\Gamma_{\rho}$, has been studied in various models. Although most of them suggest that these quantities will vanish at $T_{c}$ in the chiral limit, still it is a subtle problem to get information about the behaviour of $\Gamma_{\rho} / m_{\rho}$. In the following we will discuss some of the theoretical information about the temperature dependence of the pion and rho masses as well as $\Gamma_{\rho} / m_{\rho}$ ratio.

At zero temperature the properties of the low laying meson masses and their widths can be understood in terms of current algebra and PCAC. At low temperature, $T \ll T_{c}$, these quantities have been studied in chiral perturbation theory as well as finite temperature QCD sum rules. In $O\left(T^{2}\right)$ these calculations yield that hadron masses do not depend on the temperature in the chiral limit, while the chiral condensate drops slightly*. These investigations are in accordance with lattice QCD calculations, which do not lead to any significant temperature dependence for the hadron masses in the temperature regime studied so far, i.e. up to $T / T_{c} \simeq 0.75$. From the experimental point of view, the most relevant temperature regime is, however, close to $T_{\mathfrak{c}}$. In a heavy ion experiment

\footnotetext{
* In fact up to this order in temperature the Gell-Mann, Oakes, Renner (GMOR) relation [23] is found to be still valid [24]
} 
the hadronic medium is expected to decouple at temperatures $T_{f} \simeq 100 \mathrm{MeV}$ (freeze-out). At temperatures in the vicinity of $T_{c}$ the universality arguments may be used to predict the temperature dependence of various hadronic parameters as well as $\langle\bar{\Psi} \Psi\rangle_{T}[15,25,26]$.

So far lattice simulations of finite temperature QCD with light quarks are in accordance with the expectation that the phase transition is controlled by chiral properties of the QCD Lagrangian and that the behaviour of thermodynamic quantities in the vicinity of $T_{c}$ can be understood in terms of the dynamics of an effective threedimensional chiral Lagrangian. Although still not well established, these calculations suggest that in the limit of two vanishing quark masses $\left(m_{u}=m_{d}=0\right)$ the phase transition could be second order. In that case, the critical behaviour is expected to be governed by the fixed point of the $O(4)$ Heisenberg model in three dimensions ${ }^{\star}$. This leads to predictions for the functional form of various thermodynamic quantities in the vicinity of $T_{\mathfrak{c}}$, which all emerge from the scaling form of the singular part of the free energy [26],

$f_{s}\left(t, m_{q}\right)=b^{-1} f_{s}\left(b^{1 /(1-\delta) \beta} t, b^{\delta /(1-\delta)} m_{q}\right)$.

Here $m_{q}$ denotes the quark mass, $t=\left(T_{c}-T\right) / T_{c}$ is the reduced temperature, $\beta$ and $\delta$ are critical exponents and $b$ is an arbitrary scale parameter. Choosing either $b=m_{q}^{(\delta-1) / \delta}$ or $b=t^{(\delta-1) \beta}$ one obtains for the chiral condensate the scaling behaviour in the vicinity of the critical point $\left(t, m_{q}\right)=(0,0)$,

$\langle\bar{\Psi} \Psi\rangle_{T} \sim \begin{cases}t^{\beta}, & t>0, m_{q}=0, \\ m_{q}^{1 / \delta}, & t=0, m_{q}>0,\end{cases}$

with critical exponents of the $O(4)$ symmetric three dimensional Heisenberg model, i.e. $\beta=0.38(1)$ and $\delta=4.82(5)$. For non-vanishing $m_{q}$ and $t \neq 0$ the behaviour of $\langle\bar{\Psi} \Psi\rangle_{T}$ can be analyzed in a systematic $\varepsilon$-expansion. This has been done recently by Rajagopal and Wilczek [15]. They also use unviersality arguments to determine the behaviour of $m_{\pi}$ and $m_{\rho}$ in the vicinity of $T_{c}$. For the pion mass one finds

$m_{\pi}^{2} \sim \frac{m_{q}}{\langle\bar{\Psi} \Psi\rangle_{T}}$.

For non-vanishing quark masses the pion mass thus is expected to increase in the vicinity of $T_{c}$. This is also expected from chiral perturbation theory at $\mathcal{O}\left(T^{2}\right)$ and is known to occur also in a baryon rich matter at low densities due to the nucleon exchange [3]. We note, that the pion mass will take on a finite value at $T_{c}$.

For the discussion of dilepton spectrum, (2.4), not only $m_{\pi}$ but also $m_{\rho}$ and the ratio $\Gamma_{\rho} / m_{\rho}$ are of interest. In [15] the behaviour of $m_{\rho}$, defined as the peak of $\rho$-channel susceptibility, has been analyzed for $T>T_{c}$. It has been noted there is a link to the critical behaviour at $T_{c}$, which leads to a temperature dependent non-analytic contribu-

\footnotetext{
* The relevant symmetry group is expected to be $S U(2) \times S U(2)$, which is isomorphic to $O(4)$. If the axial $U(1)$ also gets restored at $T_{c}$, the relevant symmetry group will actually be $U(1) \times S U(2) \times S U(2)$, which is isomorphic to $O(2) \times O(4)$. In this case a 1st order transition would be likely
}

tion to the rho meson mass. However, the entire temperature dependence of $m_{\rho}$ is at present theoretically not under control. Moreover, a corresponding analysis below $T_{\mathfrak{c}}$ does, at present, not exist.

Recently in [6] by extending zero temperature sum rule relations to non-vanishing temperature the $m_{\rho}$ mass was found to decrease with temperature. In [3] it has been argued that $m_{\rho}(T) \sim\langle\bar{\Psi} \Psi\rangle_{T}{ }^{1 / 3}$. The sum rules being very successful at low temperature can, however, not describe correctly the functional dependence of hadron parameters in the vicinity of $T_{c}$. In view of this uncertain situation, we have decided to use a quite general ansatz for the temperature dependence of $m_{\rho}$,

$$
\frac{m_{\rho}(T)}{m_{\rho}(T=0)}=c\left(\frac{\langle\bar{\Psi} \Psi\rangle_{T}}{\langle\bar{\Psi} \Psi\rangle_{T=0}}\right)^{A} .
$$

The temperature dependence of $\Gamma_{\rho} / m_{\rho}$ we parameterize as

$$
\left(\frac{\Gamma_{\rho}}{m_{\rho}}\right)_{T}=c^{\prime}\left(\frac{\Gamma_{\rho}}{m_{\rho}}\right)_{T=0}\left(\frac{\langle\bar{\Psi} \Psi\rangle_{T}}{\langle\bar{\Psi} \Psi\rangle_{T=0}}\right)^{\Delta^{\prime}} \text {. }
$$

where $c$ and $c^{\prime}$ are constants, which are apriori not determined by the scaling arguments. Later we will discuss their significance and specific choice. The parameters $\Delta$ and $\Delta^{\prime}$ can in general be related if basic PCAC relations of zero temperature QCD remain valid close to $T_{c}$. In particular, when the Gell-Mann, Oakes, Renner formula [23]

$m_{\pi}^{2} f_{\pi}^{2}=2\left(m_{u}+m_{d}\right)\langle\bar{\Psi} \Psi\rangle$,

and KSFR relation [27]

$g_{\rho \pi \pi}=\frac{m_{\rho}}{\sqrt{2 f_{\pi}}}$

are valid at finite temperature, then from (2.3) one finds $\Delta^{\prime}=2 \Delta-2$. A determination of $\Delta$ and $\Delta^{\prime}$, thus would check their applicability at finite $T$. In the following, however, we will allow an independent variation of $\Delta$ and $\Delta^{\prime}$.

\section{Thermal dilepton rates in expanding QCD matter}

Let us first discuss qualitatively the modifications of the dilepton spectrum, which we can expect to result from the temperature dependence of hadronic parameters. We have calculated the dilepton spectrum at a temperature of $120 \mathrm{MeV}$, assuming for the critical temperature a value $T_{c}=150 \mathrm{MeV}$, which is suggested by recent lattice calculations [28]. It is known from lattice simulations in quenched QCD as well as 4-flavour QCD that the chiral condensate drops by more than $30 \%$ at $T_{c}$ even in these cases, where the phase transition is first order. It thus does not seem to be unreasonable to expect a similar change in the hadronic parameters. The solid curve in Fig. 1 shows the thermal dilepton invariant mass spectrum, calculated under the assumption that none of the hadronic parameters is temperature dependent. The dashed-dotted curve shows the influence of a $20 \%$ decrease in the rho meson mass. The shift of the resonance peak to smaller invariant mass values is clearly visible. If in addition the ratio $\Gamma_{\rho} / m_{\rho}$ decreases also by $20 \%$ the total rate increases 


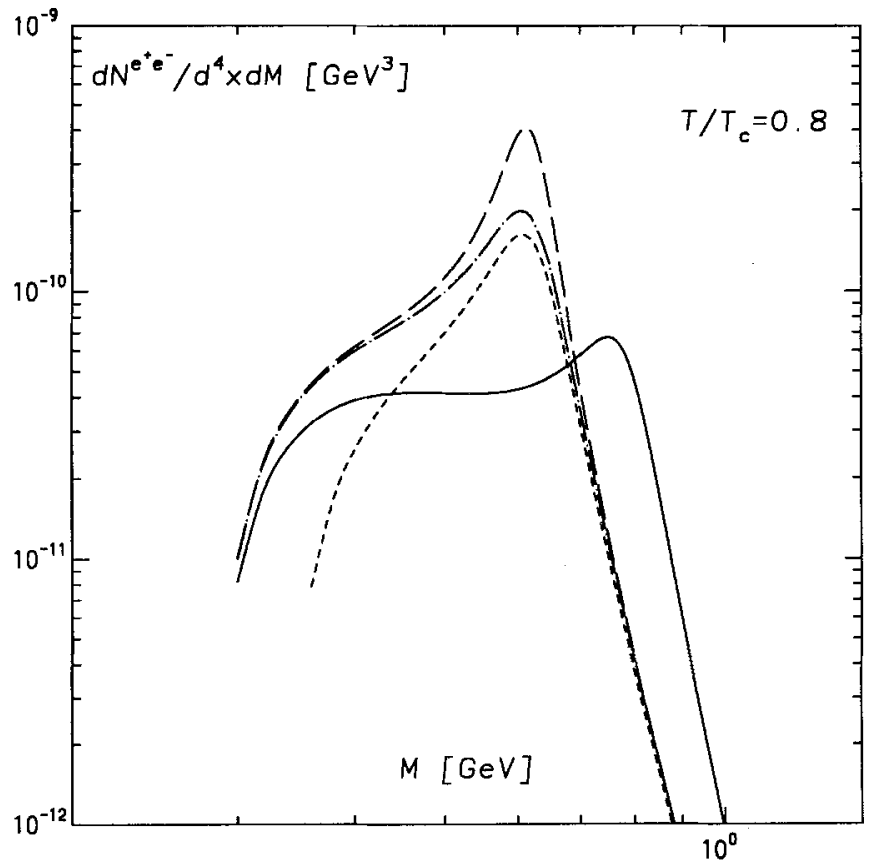

Fig. 1. Dilepton rates versus invariant mass $M$ for $T / T_{\boldsymbol{c}}=0.8$ $\left(T_{c}=150 \mathrm{MeV}\right)$. The solid curve shows the thermal spectrum assuming that none of the hadronic parameters is temperature dependent. The dashed curves show results for $m_{\rho}(T) / m_{\rho}(0)=0.8$, $\Gamma_{\rho}(T) / m_{\rho}(T)=\Gamma_{\rho}(0) / m_{\rho}(0) \quad$ (dashed-dotted), $\quad m_{\rho}(T) / m_{\rho}(0)=0.8$, $\Gamma_{\rho}(T) / m_{\rho}(T)=0.8 \Gamma_{\rho}(0) / m_{\rho}(\mathrm{G}) \quad$ (long-dashed), $\quad m_{\rho}(T) / m_{\rho}(0)=0.8$, $\Gamma_{\rho}(T) / m_{\rho}(T)=\Gamma_{\rho}(0) / m_{\rho}(0)$ and $m_{\pi}(T) / m_{\pi}(0)=1.2$ (short-dashed)

further, while the overall shape of the spectrum remains unchanged. This is shown by the long-dashed curve in Fig. 1. Finally we also illustrate the influence of a $20 \%$ increase in the pion mass (keeping $\Gamma_{\rho} / m_{\rho}$ fixed). This mainly influences the phase space cut-off in the low invariant mass region of the spectrum (short dashes).

The appearance of the shift of $\rho$-peak in the dilepton spectrum at fixed temperature was previously also indicated in [6]. There, however, the values of $I_{\rho}$ and $m_{\rho}$ at a given temperature were consistently calculated within a new formulation of QCD sum rules at finite temperature.

A further complication in the description of dilepton spectra arises from the fact that in a heavy ion collision dilepton pairs are emitted from a rapidly cooling medium rather than a medium at fixed temperature. Hot hadronic matter produced in heavy ion collisions undergoes hydrodynamic expansion leading to a space-time dependence of temperature and volume. In the expanding system we still have to integrate (2.4) over the space-time history of the collision. For this we need to assume a model for the expansion dynamics. We shall adopt here the Bjorken model for longitudinal hydrodynamical expansion. In this model all thermodynamical parameters are only a function of proper time, $\tau$, and do not depend on the rapidity variable, $y$. In the case of $1+1$ dimensional expansion the entropy current conservation implies that

$s(\tau) \tau=$ const,

with $s$ being the entropy density of the system.

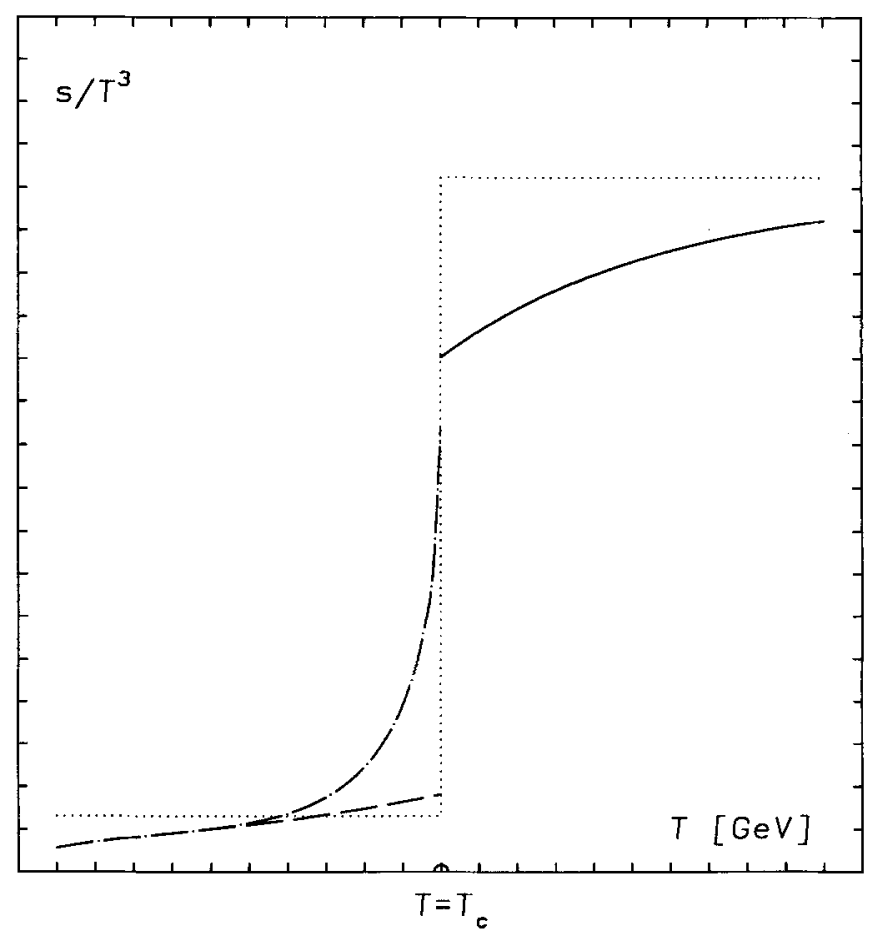

Fig. 2. Entropy density in units of $T^{3}$ versus temperature $T$. The solid curve and the dashed-dotted curve show $s / T^{3}$ as described in the text. This form has been used in the studies of the thermal dilepton spectrum. For comparison the behaviour of the entropy density in a resonance gas with temperature independent masses (dashed-curve) and ideal gases (dotted-curve) is also shown

Rather than going into details of the parameterization of the equation of state, which is not of central interest here, we show in Fig. 2 the temperature dependence of the entropy density used by us to describe the hydrodynamic expansion according to (4.1). We just mention that we have modeled the hadronic phase with a resonance gas, where we allow for temperature dependent hadron masses. Compared to the moderate rise of the entropy density in a resonance gas with temperature independent masses (dashed curve) this leads to a rather rapid rise of the entropy density close of $T_{c}$ (dashed-dotted curve). In the plasma phase we used a cut-off model [29], which extends the structure of the entropy density observed in lattice calculations of purely gluonic QCD [30] to the case of QCD with light quarks. This accounts for the drop in $s / T^{3}$ in the plasma phase close to $T_{c}$. We note that the entropy density used by us seems to be appropriate to describe the behaviour in the vicinity of a second order or weakly first order phase transition. A consequence of the rapid change of the entropy density in the vicinity of $T_{c}$ is that the system remains for quite a long time at temperatures close to $T_{c}$.

With this explicit form for the temperature dependence of the entropy density in QCD matter and (4.1) the time dependence of the relevant thermodynamic variables, in particular the temperature, can be determined. Consequently, the space-time integration in (2.4) can be performed leading to the spectrum which might be experimentally observed in heavy ion collisions. Within the expansion model adopted here, we assume, that at some 


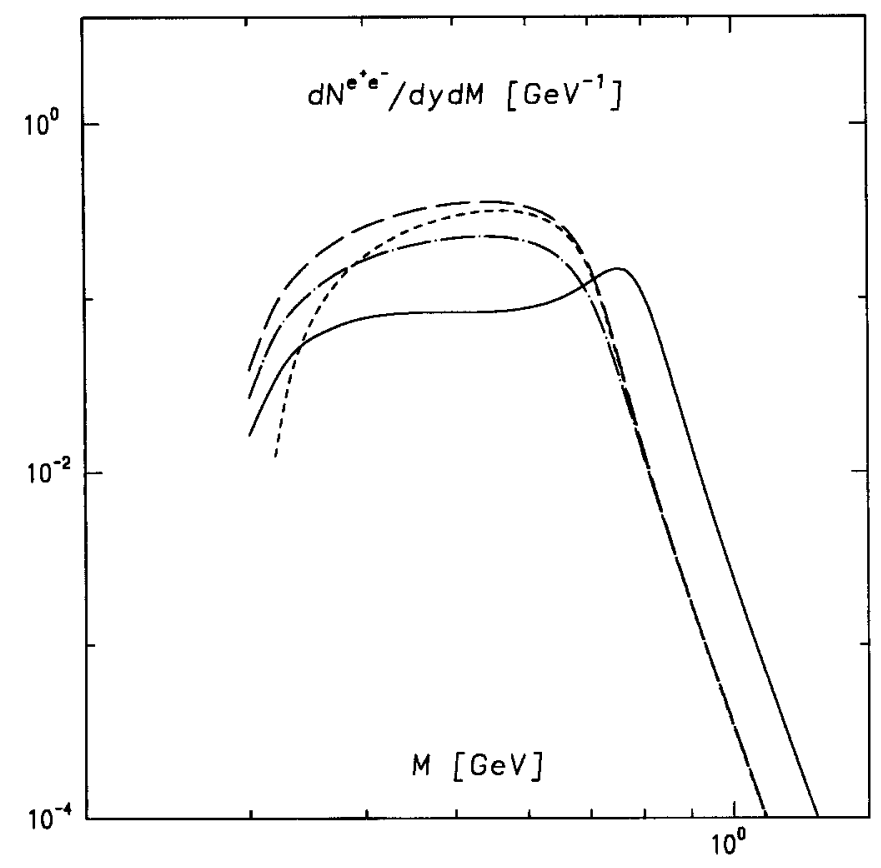

Fig. 3. Dilepton rates versus invariant mass $M$ integrated over the time history of expanding QCD matter. The temperature dependence of $m_{\rho}$ and $\Gamma_{\rho} / m_{\rho}$ has been parametrized according to (3.4) and (3.5) with $c, c^{\prime}$ chosen such that the values of Fig. 1 are obtained at $T / T_{c}=0.8$. The curves correspond to $\Delta=1, \Delta^{\prime}=0$ (dashed-dotted), $\Delta=1, \Delta^{\prime}=1$ (long-dashed) with fixed pion mass and $\Delta=1, \Delta^{\prime}=0$ assuming a temperature dependent pion mass that rises quadratically with temperature up to $m_{\pi}\left(T_{c}\right)=1.5 m_{\pi}(T=0)$ (short-dashed)

initial time $\tau_{0} \sim 1 \mathrm{fm}$ the QCD matter is formed as a thermalized quark-gluon plasma with the temperature $T_{0} \sim 0.25 \mathrm{GeV}$. The system subsequently cools until it reaches a critical temperature, $T_{c} \sim 0.15 \mathrm{GeV}[28]$. At $T=T_{c}$ the system hadronizes and stays in the hadronic phase up to a freeze-out temperature $T_{f} \sim 100 \mathrm{MeV}$.

As has been shown in Fig. 1 the temperature dependence of $\Gamma_{\rho} / m_{\rho}$ as well as the pion mass plays a minor role for the structure of the dilepton spectrum. In fact, the modifications of the spectrum due to these effects, which are visible in Fig. 1, reduce even further when we integrate the dilepton distributions over the time history of the hot hadronic medium. This is shown in Fig. 3 for various values of $\Delta$ and $\Delta^{\prime}$. The main effect comes from the temperature dependence of the rho mass. It leads to a smearing of the shifted rho peak over a wide range of invariant mass values. We also note that an extrapolation of the low temperature results from chiral perturbation theory up to $T_{c}$ would at most suggest an increase of $m_{\pi}$ by $5 \%$ [24]. Temperature effects on the pion mass are thus not expected to lead to significant modifications of the dilepton spectrum.

A further characteristic feature of the distributions is the shift of the exponentially decaying part at large invariant mass to smaller values. This is related to the value of $m_{\rho}\left(T_{f}\right)$, i.e. the rho mass at freeze-out. In the parameterization given by (3.4) this can either be controlled by changing $\Delta$ or the proportionality constant $c$. Furthermore, also a change in the actual value of the freeze-out temperature clearly influences the value of $m_{\rho}\left(T_{f}\right)$. The relevance of this

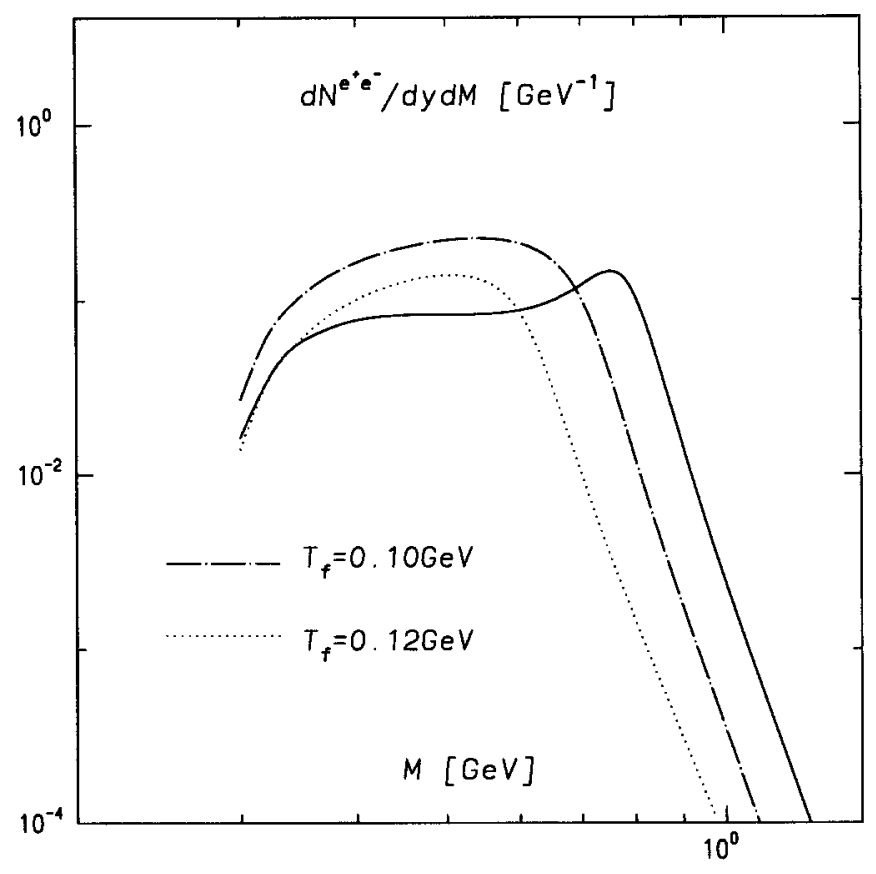

Fig. 4. Dilepton rates versus invariant mass $M$ integrated over the time history of expanding QCD matter. Shown is the dependence of the thermal dilepton rate on the freeze-out temperature assuming $m_{\pi}$ and $\Gamma_{\rho} / m_{\rho}$ to be temperature independent and $m_{\rho}(T)$ to vary according to (3.4) with $\Delta=1$ and $m_{\rho}(T=100 \mathrm{MeV})=m_{\rho}(0)$. The solid curve gives the result for temperature independent hadronic parameters

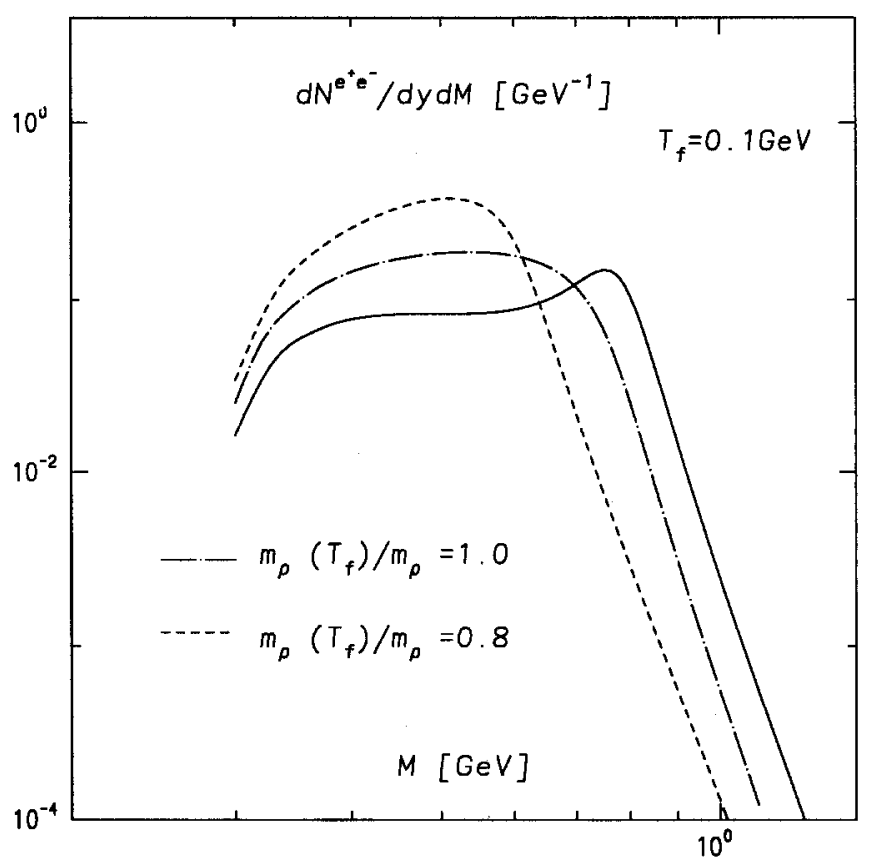

Fig. 5. Same as Fig. 4, however, at fixed freeze-out temperature, $T_{f}=100 \mathrm{MeV}$, and different values for the rho mass at freeze-out as given in the figure

is illustrated in Figs. 4 and 5, where we show the invariant mass dilepton spectrum, assuming two different values for the freeze-out temperature (Fig. 4) with $c$ in (3.4) chosen such that $m_{\rho}\left(T_{f}=100 \mathrm{MeV}\right)=m_{\rho}(T=0)$ as well as two 


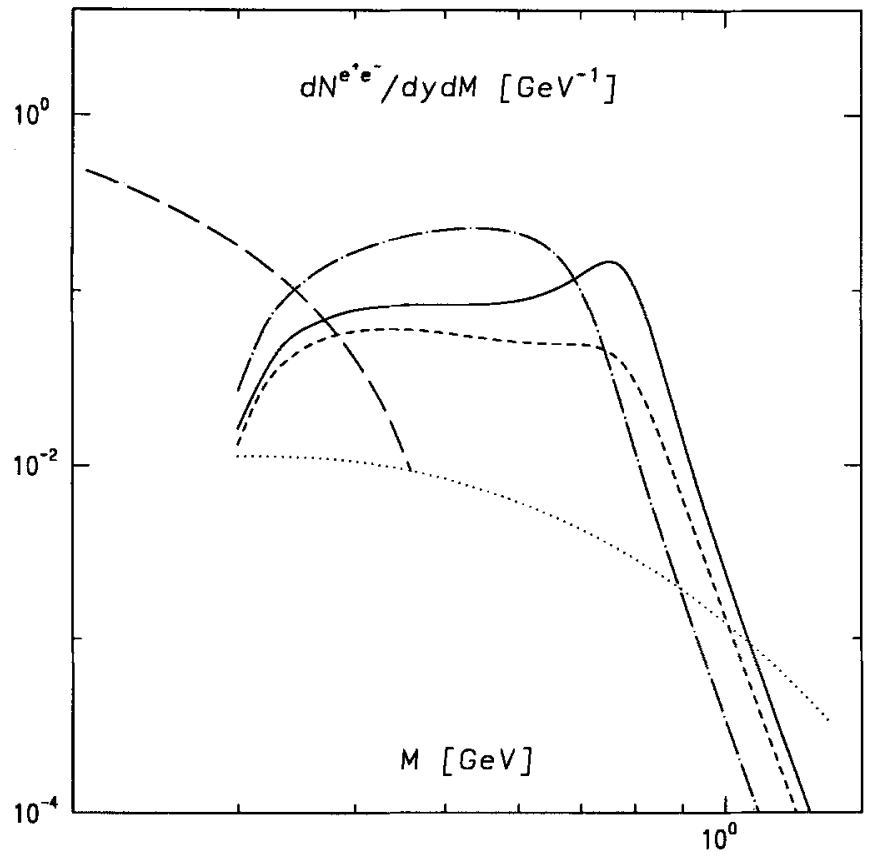

Fig. 6. Summary of our results for the thermal dilepton rates. The full line and the dashed-dotted curve are taken from Fig. 5. The short-dashed curve shows the result of [5]. Also shown is the background from Dalitz decays of $\eta^{\prime}$ s (long-dashed curve) and thermal dileptons from the quark-gluon plasma (dotted curve)

different values for $m_{\rho}\left(T_{f}\right) / m_{\rho}$ (Fig. 5) for fixed value of the freeze-out temperature. We note that in all cases the integration over the time history of the hadronic matter results in a broadening of the dilepton spectrum, which wipes out the rho resonance peak completely.

Finally we compare the thermal dilepton spectrum with background from Dalitz $\eta$ decays. This is shown in Fig. 6. We note that the thermal distribution should be visible well above the background from Dalitz decays. A comparison with Fig. 1 also shows that it is unlikely to observe variations of the low mass cut-off in the thermal spectrum due to changes in the pion mass.

\section{Conclusions}

The production rate of dileptons provides one of the main tools for the study of a quark-gluon plasma formation in heavy ion collisions. We have analyzed the implications of the chiral symmetry restoration phase transition on dilepton production in a thermal hadronic medium. In this analysis, we have adopted a simplified $(1+1)$-dimensional Bjorken model to describe the expansion of the system. We have further used an equation of state of QCD matter which effectively takes into account the non-perturbative effects, which are known to be important in the vicinity of the QCD phase transition.

It is clear from our considerations that the dilepton mass distribution integrated over the space time history of the collision, depends on various external parameters in addition to the temperature dependent hadronic parameters: the initial thermalization time, $\tau_{0}$, the initial $\left(T_{0}\right)$, the critical $\left(T_{c}\right)$ and the freeze-out $\left(T_{f}\right)$ temperatures. We have used conservative values for these parameters which are adequate for $\mathrm{U}-\mathrm{U}$ collisions at $\mathrm{LHC}$ energy $\left(\tau_{0} \sim 1 \mathrm{fm}\right.$, $T_{0} \sim 0.25 \mathrm{GeV}, T_{c} \sim 0.15 \mathrm{GeV}$ and $T_{f} \sim=0.1 \mathrm{GeV}[31]$ ). Arguments have been given that the initial entropy density of the quark-gluon plasma for LHC energy could be even of the order of $1.0 \mathrm{GeV}$ [32]. The resulting increase of the initial temperature would influence the quark-gluon plasma contribution to the over-all dilepton production rate. However, it will not change our discussion on the hadronic contribution.

The transverse collective flow would certainly modify the details of our results. However, for a comparison of dilepton rates with and without the chiral modification of the hadron spectrum, the transverse expansion is not of crucial importance. It would influence the "vacuum" and "medium" results in a similar way leaving the relative change almost unaffected.

The influence of the chiral phase transition on the hadron spectrum is still not well established. Thus, in our discussion, we have concentrated on basic features expected from various theoretical model calculations. As a basis for our parameterizations we have assumed the existence of a second order chiral transition at which the rho meson mass vanishes. We have shown, that the temperature variation of hadron parameters, due to the chiral phase transition, leads to significant modifications of the dilepton production rates even if these parameters deviate from the corresponding zero temperature values only in the vicinity of $T_{c}$. The specific structure of the dilepton spectrum will depend on details in the temperature dependence of the hadronic parameters as well as the time evolution of the hadronic medium. Nevertheless, independent of such details a common feature emerging from the temperature dependence of the rho mass is the smearing of the spectrum, which completely eliminates the thermal rho peak. Even if the rho meson mass does not vanish at $T_{c}$ but would drop only by $\sim 20 \%$ such a broadening should occur as can be deduced already from Fig. 1.

We thus expect that a detailed experimental study of the dilepton spectrum will yield important information about the temperature dependence of hadronic parameters in the vicinity of the chiral phase transition. Further lattice calculations of the hadron spectrum at temperatures closer of $T_{c}$ should also help to clarify this issue.

Acknowledgments. We acknowledge very interesting discussions with S. Gupta, E. Laermann, B. Petersson and H. Satz. One of the authors (K.R) is also indebted to H. Satz for his kind hospitality at the University of Bielefeld. This work was partly supported by the Bundesministerium für Forschung und Technologie (BMFT) and the DFG under contract Pe 340/1-3.

\section{References}

1. R. Pisarski: Phys. Lett. B110 (1982) 155

2. C.M. Ko, Z.G. Wu, G.E. Brown: Phys. Rev. Lett. 66 (1991) 2577; C.M. Ko, B.H. Sa: Phys. Lett. B258 (1991) 6

3. G. Brown: Nucl. Phys. A522 (1991) 397c

4. Bi Pin-Zhen, J.Rafelski: Mod. Phys. Lett. A27 (1992) 2493

5. C. Dominguez: Z. Phys, C 49 (1991) 423

6. T. Hatsuda, Y. Koike, Su H. Lee: Nucl. Phys. B394 (1993) 221 
7. C. Gale, J.I. Kapusta: Nucl. Phys. B357 (1991) 65

8. H. Leutwyler, A.V. Smilga: Nucl. Phys. B342 (1990) 302

9. T. Kunihiro: Nucl. Phys. B351 (1991) 593

10. C. Adami, T. Hatsuda, I. Zahed: Phys. Rev. D43 (1991) 921

11. S. Gupta: Phys. Lett. B288 (1992) 171; A. Gocksch, P. Rossi, U.M. Heller: Phys. Lett. B205 (1988) 334; A. Gocksch: Phys. Rev. Lett. 67 (1991) 1701; K. Born et al.: Phys. Rev. Lett. 67 (1991) 302; V.L. Eletskii, B.L. Ioffe: Sov. J. Nucl. Phys. 48 (1988) 384

12. Su H. Lee: Phys. Rev. D40 (1989) 3484; R.J. Furnstahl, T. Hatsuda, Su H. Lee: Phys. Rev. D42 (1990) 1744

13. G.E. Brown, M. Rho: Phys. Rev. Lett. 66 (1991) 2720

14. C. Adami, G.E. Brown: Phys. Rev. D46 (1992) 478

15. F. Wilczek: Int. J Mod. Phys. A7 (1992) 3911; K. Rajagopal, F. Wilczek: PUPT-1347, IASSNS-HEP-92/60

16. For a recent reviews see: P.V. Ruuskanen: in: Proceedings of QM'91, Nucl. Phys. A544 (1992) 371c, T.C. Awes et al. (eds.); H. Satz: Proc. Int. Lepton-Photon Symp., Geneva, Switzerland (1991); Nucl. Phys. A525 (1991) 255c

17. H. Satz: in: Proceedings of the ECFA Workshop on Large Hadron Colliders, Aachen, Germany, 1990, G. Jarlskog, D. Rein (eds.) (CERN Report No. 90-10, Geneva, 1990) Vol. I, p. 188; in: Proceedings of QM'91, Nucl. Phys. A544 (1992) 371c
18. J. Cleymans, K. Redlich, H. Satz: Z. Phys. C 52 (1991) 517

19. J. Cleymans, V.V. Goloviznin, K. Redlich: Phys. Rev. D47 (1993) 173; J. Cleymans, V.V. Goloviznin, K. Redlich: Phys. Rev. D47 (1993) 989

20. K. Haglin, C. Gale, V. Emel'yanov: Phys. Rev. D47 (1993) 973

21. P.V. Ruuskanen: Acta. Phys. Pol. B18 (1986) 551

22. G. Domokos: Phys. Rev. D28 (1983) 123

23. M. Gell-Mann, R.J. Oakes, B. Renner: Phys. Rev. 175 (1968) 2195

24. J. Gasser, H. Leutwyler: Phys. Lett. B184 (1987) 83

25. R.D. Pisarski, F. Wilczek: Phys. Rev. D29 (1984) 338

26. G. Boyd et al.: Nucl. Phys. B376 (1992) 199

27. K. Kawarabyashi, M. Suzuki: Phys. Rev. Lett. 16 (1966) 255

28. S. Gottlieb et al.: Phys. Rev. D35 (1987) 3972; R.V. Gavai et al.: Phys. Lett. B241 (1990) 437

29. J. Engels et al.: Z. Phys. C42 (1989) 341

30. J. Engels et al:: Phys. Lett. B252 (1990) 625

31. H. Satz: in: Proceedings of ECFA Large Hadron Collider Workshop, Aachen 1990; Volume I, CERN (1990)

32. J. Kapusta, L. McLerran, D. Kumar Srivastava: Phys. Lett. B283 (1992) 145 